\title{
Rice functional genomics: theories and practical applications
}

\author{
Lizhong Xiong • Yusaku Uga • Yibo Li
}

Published online: 18 July 2020

(C) Springer Nature B.V. 2020

Rice is not only a staple food crop worldwide but also a key model cereal crop for genomic and plant science research. Rice has made great progress in the last three decades in functional genomics and genetic improvement, playing a leading role among crops. Aiming to realize less pesticide, less chemical fertilizer, water saving and drought resistance, and acceptable grain quality and high yield in rice production, Green Super Rice (GSR), proposed by Prof. Qifa Zhang (Huazhong Agricultural University, China), provides a promising strategy for sustainable agriculture and will be boosted by the breakthrough and systematical achievements of rice functional genomics. Thus, the Editor-in-Chief of Molecular Breeding, Prof. Qifa Zhang, invited Prof. Lizhong Xiong (Huazhong Agricultural University, China) and Prof. Yusaku Uga (National Agriculture and Food Research Organization, Japan) as two guest editors to host the special issue named "Rice Functional

This article is part of the Topical Collection on Rice Functional Genomics

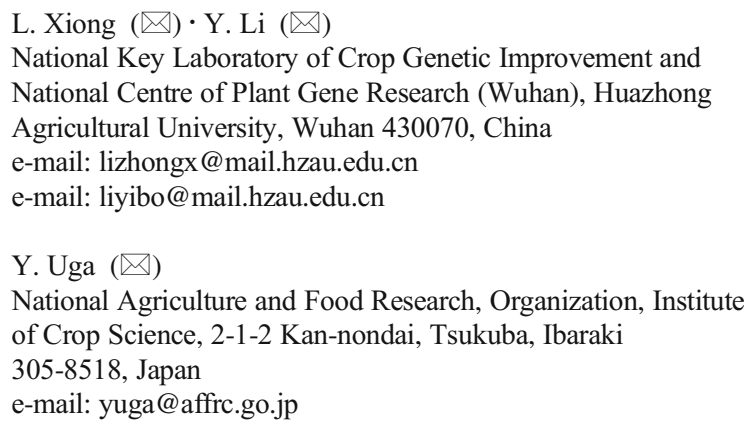

Genomics." In this special issue, we invited a number of experts from major rice production countries to present the advances of rice functional genomics research and offer their perspectives for future developments and practical applications in rice molecular breeding. There are 13 papers published in this issue covering various aspects, including 5 main topics: yield capacity, nutrition use efficiency, biotic and abiotic stress resistance, and grain quality, which are vital agronomic traits for rice production. Here, we summarize some of the highlights from these papers.

\section{Yield capacity}

Source capacity in crops refers to the availability of a carbon supply to meet the demand from the sink organs. Source capacity is determined by photosynthesis capacity, leaf senescence, canopy architecture, and translocation. However, there is a big gap between the basic studies of source capacity and practical breeding programs to increase yield. Adachi et al. (2020) summarized the physiological and genetic factors that regulate source capacity gained by transgenic approaches. They also highlighted the development of phenotyping methods that can be used to promote genetic analysis of source capacity and our current understanding of the genetic controls of source capacity underlying natural variations. They finally presented three major challenges that must be solved to enhance source capacity and increase grain productivity in rice. 
Final grain size and grain number per plant, which constitute the sink capacity of rice and other cereals, are two important traits determining grain yield. These two major components of sink strength generally constrain each other due to a negative correlation between grain size and grain number per panicle. The article entitled Molecular, cellular, and Yin-Yang regulation of grain size and number in rice by Fan and $\mathrm{Li}$ (2019) provided a comprehensive and systematical overview of the recent researches and future perspectives in coordinating grain size and number in rice, which has substantial implications for breaking the yield bottleneck caused by the trade-off of grain size and number in crop genetic improvement. Grain size and number are determined by the size, activity and number of inflorescence, and branch and floral meristems, which are coordinately regulated by both rate and duration of cell division, expansion, and differentiation. They summarized nine signaling pathways controlling grain size and number, including $\mathrm{G}$ protein signaling; biosynthesis and signaling of BR, CK, and IAA; MAPK signaling; peptide signaling; the ubiquitin-proteasome degradation pathway; epigenetic pathways; and transcriptional regulation. They also proposed 16 key scientific questions underlying both applied and basic research of grain size and number in rice and other crops to call for an international coordinated effort in solving them in the form of a project named GSGN16.

To meet the increasing demands in crop production, improving plant architecture, which consists mainly of plant height, tillering, and panicle branching, is often used as an effective strategy to promote yield. In recent years, great progress has been made in exploring the molecular mechanisms of rice plant architecture, which greatly improves our ability to rationally modify rice architecture. In addition, plants have remarkable plasticity by modifying their morphology in response to various environmental factors. In this review entitled Molecular mechanisms underlying plant architecture and its environmental plasticity in rice, Gao et al. (2019) emphasized recent progress in elucidating the molecular mechanisms that control rice plant architecture, focusing on tiller number, tiller angle, and panicle branching. They also discussed the roles of various environmental factors in influencing the plasticity of rice plant architecture, although more studies may be needed to illustrate the crosstalk mechanisms between rice plant architecture and environment.
The root system architecture (RSA) is crucial for plants to survive through taking up nutrients and water from the soil and adapt to environmental stresses, and thus is an important breeding target for developing climate-resilient rice. Because roots are hidden underground, high-throughput root phenomics has not been established not only in rice but also in whole plants, which represents a bottleneck for employing a multiomics approach for accelerating molecular breeding of RSA. Yoshino et al. (2019) reviewed previous transcriptome analyses for root development under various abiotic stresses such as drought, salinity, and heat and assessed the recent status of high-throughput root phenotyping and modeling technologies in rice, which are expected to be effective for accelerating molecular breeding. Finally, they discussed the application of these technologies for functional genomics of RSA and its molecular breeding that is robust under various environmental stresses.

\section{Nutrition use efficiency}

Phosphorus (P) is an essential macronutrient for plant growth and development. However, only $10-25 \%$ of the applied Pi fertilizer is taken up by crops. Overuse of $\mathrm{P}$ fertilizer to increase food production for feeding the growing population causes a series of environmental problems, such as accelerating water eutrophication and soil degradation. Therefore, it is urgent to improve crop $\mathrm{P}$ use efficiency to reduce the use of Pi fertilizers and their escape in the environment for sustainable agriculture. The review by He et al. (2019) provided a comprehensive overview of current progresses on molecular mechanisms and regulatory pathways involved in rice root $\mathrm{Pi}$ acquisition from soil, Pi transportation from root to shoot, and allocation, utilization, and remobilization of Pi within plant shoot tissues. They also outlined the current challenges and possible strategies for improving crop P use efficiency.

A deficiency or an excess of micronutrients such as iron $(\mathrm{Fe})$, copper $(\mathrm{Cu})$, manganese $(\mathrm{Mn})$, and zinc $(\mathrm{Zn})$ severely impairs plant growth and normal development. Problems in acquiring essential minerals in plants impair not only plant growth and yield but also significantly affect human and animal health. Plants utilize extremely complex and tightly regulated mechanisms to acquire these minerals from soil, transport them from roots to shoots, and deliver them 
to developing tissues and edible parts of plants. In the paper entitled The transport of essential micronutrients in rice, Bashir et al. (2019) updated recent understanding of metal transport in plants with a particular focus on rice, mainly including acquisition of micronutrients from the rhizosphere, distribution of metals within the plant body, subcellular metal homeostasis, and potential of plant transporters for improvement of crop plants. They also discussed strategies for breeding crops that efficiently regulate the uptake and storage of these metals suitable for a diverse range of soils and climates, which will contribute to the production of healthier food for human consumption.

\section{Abiotic stress resistance}

Rice production consumes approximately $55 \%$ of China's total freshwater resources. The environmental and resource challenges faced by rice production call for environment-friendly and resource-saving rice varieties. Water-saving and drought-resistant rice (WDR) is a new type of cultivated rice combining both high yield potential and acceptable grain quality as a current lowland paddy rice, as well as water-saving and drought resistance as a traditional upland rice. Twenty-two WDR varieties were registered and distributed to farmers in recent years in China and could be planted in both rainfed and irrigated ecosystems. In the paper entitled Water-saving and drought-resistant rice: from the concept to practice and theory, Luo et al. (2019) systematically reviewed the genetic and physiological mechanisms of rice adaptation to drought, differentiation of drought resistance among lowland-upland rice germplasm, and tradeoff between yield potential and drought resistance resulting in bidirectional selection during domestication of upland rice. They then outlined the concept, development, management technology, and perspective of WDR.

Soil salinity is widespread in crop-producing areas globally, restricting both vegetative growth and grain yield of crops. Due to the lack of fresh water and reduction of cultivated land, it is encouraged to use saline-alkali land to make up for food shortages. The paper by Qin and Huang (2020) provided a comprehensive overview of the recent research on identifying the components and pathways involved in rice adaption to salt stress and the approaches that can be used for breeding and cultivating salt-tolerant rice. They especially pointed out the potential phytohormonal regulation of the components and the homeostasis of $\mathrm{Na}^{+} /$ $\mathrm{K}^{+}$and reactive oxygen species.

Although water is essential for plant growth, excess water due to flooding has adverse effects on the growth of most plants because it limits oxygen and carbon dioxide exchange and reduces light intensity. To overcome these challenges, aquatic and semiaquatic plants have evolved and developed unique strategies that allow them to thrive under excess water conditions. Kuroha and Ashikari (2020) described recent progress in the understanding of the various molecular mechanisms and genetic factors regulating flooding tolerance in rice. They presented specialized anatomical and morphological adaptations to conditions of excess water in rice, including aerenchyma formation, radial oxygen loss barrier, adventitious root emergence, and leaf gas film formation ability. They also outlined strategies to adapt to complete submergence in rice, including molecular mechanisms of anaerobic germination tolerance, flash flood tolerance, SUB1Amediated quiescent strategy, tolerance to periodic flooding, hormonal regulation as an escape strategy, and evolutionary history of deep-water rice.

In general, rice production is sensitive to temperature fluctuations and the optimum temperature for rice cultivation ranges from 25 to $30{ }^{\circ} \mathrm{C}$. Thus, basically as a tropical crop, rice is in general vulnerable to the adverse effects of low temperature. To cope with or adapt to cold stress condition, rice has evolved elaborate regulatory mechanisms under both natural and artificial selection, and its distribution also extends to subtropical and temperate regions. The paper entitled Current understanding of genetic and molecular basis of cold tolerance in rice by Lv et al. (2019) updated the achievements of cold tolerance-related quantitative trait loci identified by linkage and association mapping, together with key genes involved in cold sensing, signal transduction, and response. They also highlighted the progress in cold tolerance improvement using genomic breeding strategy.

\section{Biotic stress resistance}

A large variety of pathogens cause rice bacterial, fungal, viral, and nematode diseases with various symptoms on different rice tissues, which greatly affect grain yield and quality. Pyramiding resistance quantitative trait loci 
or genes into rice cultivars is an operable and optimal strategy for rice disease-resistance improvement. This paper by Zhang et al. (2019) summarized the cloned major resistance genes for several major rice diseases and elucidated the underlying molecular mechanisms. They also proposed the operable strategies to apply these R genes, QTLs, and defense-response genes against various diseases to make rice cultivars exhibit broad-spectrum and durable resistance without agronomic trait penalty.

Insects pose severe constraints to agriculture and threaten food security worldwide. Insect infestations are especially severe in rice plants, which grow in warm and humid environments and provide an attractive and nutritious food source for many phytophagous insects. In the paper entitled Current understanding of the genomic, genetic, and molecular control of insect resistance in rice, Du et al. (2020) outlined great progress made in the screening of insect-resistant rice germplasm, identifying insectresistant genes, and uncovering molecular mechanisms of host resistance. They also addressed research advances underpinning strategies to improve rice resistance against insect pests and proposed many challenges to achieve reliable insect resistance in rice.

\section{Grain quality}

With improving living standards, food demand is no longer simply "enough" but is increasingly "healthy" and "delicious." Each of rice grain quality traits, including appearance quality, milling quality, cooking and sensory quality, nutrition quality, and hygiene quality, is a complex trait that reflects the opinions of producers, processors, sellers, and consumers in response to grain production, processing, marketing, and consumption. To meet the needs of producers and consumers, researchers have to understand the molecular genetic basis that determines rice grain quality, and breeders and seed companies have to develop varieties with high yield, good quality, and health benefits. The review by Zhou et al. (2019) summarized consumer demand for rice quality, common and regional characteristics of good-quality rice, classification and importance of rice quality, and increasing health awareness of consumers. They also provided a comprehensive overview on the functional genes for various grain quality traits in rice and the genetic improvement practices of grain quality, and then discussed challenges and prospects of rice grain quality improvement.

Finally, it is worth to note that because of space limitation and the wide diversity of the rice research, this special issue only presents a very modest sample for the vast scale of rice functional genomics research in such a special issue. We thank the Editor-in-Chief, Professor Qifa Zhang, for initiating this subject to promote rice science research and especially thank Professors Lizhong Xiong and Yusaku Uga for their great efforts serving as the issue guest editors. We are also very grateful to all the contributors for sharing their knowledge and enthusiasm for preparing this special issue.

Author contribution Lizhong Xiong and Yusaku Uga were responsible for inviting and handling all the papers. Yibo Li wrote the Editorial and Lizhong Xiong and Yusaku Uga edited it.

\section{References}

Adachi S, Ohkubo S, San NS, Yamamoto T (2020) Genetic determination for source capacity to support breeding of high-yielding rice (Oryza sativa). Mol Breeding 40:20. https://doi.org/10.1007/s11032-020-1101-5

Bashir K, Seki M, Nishizawa NK (2019) The transport of essential micronutrients in rice. Mol Breeding 39:168. https://doi. org/10.1007/s11032-019-1077-1

Du B, Chen R, Guo J, He G (2020) Current understanding of the genomic, genetic, and molecular control of insect resistance in rice. Mol Breeding 40:24. https://doi.org/10.1007/s11032020-1103-3

Fan Y, Li Y (2019) Molecular, cellular and Yin-Yang regulation of grain size and number in rice. Mol Breeding 39:163. https://doi.org/10.1007/s11032-019-1078-0

Gao H, Wang W, Wang Y, Liang Y (2019) Molecular mechanisms underlying plant architecture and its environmental plasticity in rice. Mol Breeding 39:167. https://doi. org/10.1007/s11032-019-1076-2

He Q, Wang F, Wang Y, Lu H, Yang Z, Lv Q, Mao C (2019) Molecular control and genetic improvement of phosphorus use efficiency in rice. Mol Breeding 39:162. https://doi. org/10.1007/s11032-019-1059-3

Kuroha T, Ashikari M (2020) Molecular mechanisms and future improvement of submergence tolerance in rice. Mol Breeding 40:41. https://doi.org/10.1007/s11032020-01122-y

Luo L, Mei H, Yu X, Xia H, Chen L, Liu H, Zhang A, Xu K, Wei H, Liu G, Wang F, Liu Y, Ma X, Lou Q, Feng F, Zhou L, Chen S, Yan M, Liu Z, Bi J, Li T, Li M (2019) Water-saving and drought-resistance rice: from the concept to practice and theory. Mol Breeding 39:145. https://doi.org/10.1007 /s11032-019-1057-5

Lv Y, Hussain MA, Luo D, Tang N (2019) Current understanding of genetic and molecular basis of cold tolerance 
in rice. Mol Breeding 39:159. https://doi.org/10.1007 /s11032-019-1073-5

Qin H, Huang R (2020) The phytohormonal regulation of $\mathrm{Na}^{+} / \mathrm{K}^{+}$ and reactive oxygen species homeostasis in rice salt response. Mol Breeding 40:47 https://link.springer. com/article/10.1007/s11032-020-1100-6

Yoshino K, Numajiri Y, Teramoto S, Kawachi N, Tanabata T, Tanaka T, Hayashi T, Kawakatsu T, Uga Y (2019) Towards a deeper integrated multi-omics approach in the root system to develop climate-resilient rice. Mol Breeding 39:165. https://doi.org/10.1007/s11032-019-1058-4
Zhang M, Wang S, Yuan M (2019) An update on molecular mechanism of disease resistance genes and their application for genetic improvement of rice. Mol Breeding 39:154. https://doi.org/10.1007/s11032-019-1056-6

Zhou H, Xia D, He Y (2019) Rice grain quality - traditional traits for high quality rice and health-plus substances. Mol Breeding 40:1. https://doi.org/10.1007/s1 1032-019-1080-6

Publisher's note Springer Nature remains neutral with regard to jurisdictional claims in published maps and institutional affiliations. 